\title{
A Special Class of Bell Polynomials
}

\author{
By F. T. Howard
}

Abstract. We examine the integers $V(n, k)$ defined by means of

$$
k ! \sum_{n=0}^{\infty} V(n, k) x^{n} / n !=\left[x\left(e^{x}+1\right)-2\left(e^{x}-1\right)\right]^{k},
$$

and, in particular, we show how these integers are related to the Bernoulli, Genocchi and van der Pol numbers, and the numbers generated by the reciprocal of $e^{x}-x-1$. We prove that the $V(n, k)$ are also related to the numbers $W(n, k)$ defined by

$$
k ! \sum_{n=0}^{\infty} W(n, k) x^{n} / n !=\left[(x-2)\left(e^{x}-1\right)\right]^{k}
$$

in much the same way the associated Stirling numbers are related to the Stirling numbers. Finally, we examine, more generally, the Bell polynomials $B_{n, k}\left(a_{1}, a_{2}, 3-\alpha, 4-\alpha, 5-\alpha, \ldots\right)$ and show how the methods of this paper can be used to prove several formulas involving the Bernoulli and Stirling numbers.

1. Introduction. For $n$ and $k$ nonnegative integers, define $V(n, k)$ by means of

$$
k ! \sum_{n=0}^{\infty} V(n, k) x^{n} / n !=\left[x\left(e^{x}+1\right)-2\left(e^{x}-1\right)\right]^{k}=\left[\sum_{n=3}^{\infty}(n-2) x^{n} / n !\right]^{k} \text {. }
$$

Thus, $V(n, k)=B_{n, k}(0,0,1,2,3, \ldots)$, the exponential partial Bell polynomial [2, p. 133].

One purpose of this paper is to derive properties of $V(n, k)$ and, in particular, to show how the $V(n, k)$ are related to certain special sequences of numbers. For example, in Section 4 we show that if $G_{n}$ is the $n$th Genocchi number, then

$$
G_{n}=\sum_{j=0}^{n-1} j ! 4^{-j}\left(n V(n-1, j)-\left(\begin{array}{l}
n \\
2
\end{array}\right) V(n-2, j)\right),
$$

and we prove similar equations involving the Bernoulli numbers, the van der Pol numbers, and the numbers generated by the reciprocal of $e^{x}-x-1$. (All of these special numbers are defined in Section 2.) Thus, the $V(n, k)$ provide a link between these special numbers which is not obvious.

In Section 5 we look, more generally, at the Bell polynomials $3_{n, k}\left(a_{1}, a_{2}, 3-\alpha, 4-\alpha, 5-\alpha, \ldots\right)$; and we show how the results of Section 4 can

Received June 25, 1979.

1980 Mathematics Subject Classification. Primary 10 A40.

Key words and phrases. Exponential partial Bell polynomial, Stirling number of the second kind, associated Stirling number of the second kind, Bernoulli number, Genocchi number, van der Pol number. 
be generalized. Using the theorems of Section 5, we prove several of the formulas in [2], [10] and [11] involving the Bernoulli and Stirling numbers.

We begin with a preliminary section in which we state the basic definitions and theorems that we need.

2. Preliminaries. The exponential partial Bell polynomials $B_{n, k}\left(a_{1}, a_{2}, a_{3}, \ldots\right)$ in an infinite number of variables $a_{1}, a_{2}, a_{3}, \ldots$ can be defined by means of

$$
k ! \sum_{n=0}^{\infty} B_{n, k}\left(a_{1}, a_{2}, \ldots\right) x^{n} / n !=\left(\sum_{m=1}^{\infty} a_{m} x^{m} / m !\right)^{k} .
$$

Certain special cases are well known; for example, $B_{n, k}(1,1,1, \ldots)=S(n, k)$, the Stirling number of the second kind, and $B_{n, k}(0,1,1, \ldots)=S_{1}(n, k)$, the associated Stirling number of the second kind [4], [11, p. 77]. It follows that

$$
\begin{aligned}
& B_{n, k}(0,2,3,4, \ldots)=n ! S(n-k, k) /(n-k) !, \\
& B_{n, k}(0,0,3,4, \ldots)=n ! S_{1}(n-k, k) /(n-k) ! .
\end{aligned}
$$

Next we define $R_{n}\left(a_{0}, a_{1}, a_{2}, \ldots\right)$ by means of

$$
\sum_{n=0}^{\infty} R_{n}\left(0, \ldots, 0, a_{r}, a_{r+1}, \ldots\right) x^{n} / n !=\left(a_{r} x^{r} / r !\right)\left(\sum_{i=r}^{\infty} a_{i} x^{i} / i !\right)^{-1}
$$

We are assuming $r \geqslant 0$ and $a_{r} \neq 0$.

There are useful relationships between $B_{n, k}\left(a_{1}, a_{2}, \ldots\right)$ and $R_{n}\left(a_{0}, a_{1}, a_{2}, \ldots\right)$ which we state in the next theorem [2, p. 142], [6].

THEOREM 2.1. $B_{n, k}\left(a_{1}, a_{2}, \ldots\right)$ is defined by $(2.1)$ and $R_{n}\left(a_{0}, a_{1}, a_{2}, \ldots\right)$ is defined by (2.4), then

$$
\begin{aligned}
& R_{n}\left(0, \ldots, 0, a_{r}, a_{r+1}, \ldots\right) \\
& \quad=\sum_{j=1}^{n} \frac{\left(-a_{r}\right)^{-j} n ! j !(r !)^{j}}{(n+r j) !} B_{n+r j, j}\left(0, \ldots, 0, a_{r+1}, a_{r+2}, \ldots\right) \\
& \quad=\sum_{j=1}^{n} \frac{\left(-a_{r}\right)^{-j} n ! j !(r !)^{j}\left(\begin{array}{l}
n+1 \\
j+1
\end{array}\right)}{(n+r j) !} B_{n+r j, j}\left(0, \ldots, 0, a_{r}, a_{r+1}, \ldots\right) .
\end{aligned}
$$

Also useful for our purposes are theorems relating $B_{n, k}\left(0, \ldots, 0, a_{r}, a_{r+1}, \ldots\right)$ and $B_{n, k}\left(0, \ldots, 0, a_{r+1}, a_{r+2}, \ldots\right)$. There are several such theorems proved in [2] and [5]; in particular we have the following:

THEOREM 2.2. For $r \geqslant 0, k \geqslant 0$,

$$
\begin{aligned}
\left(r ! / a_{r}\right)^{n} B_{r n+k, n} & \left(0, \ldots, 0, a_{r}, a_{r+1}, \ldots\right) \\
& =(r n+k)(r n+k-1) \ldots(n+1) P_{r, k}(n),
\end{aligned}
$$


where $P_{r, k}(n)$ is a polynomial in $n$ of degree $\leqslant k$. (The degree is $k$ if $a_{r+1} \neq 0$.) In fact

$$
P_{r, k}(n)=\sum_{j=1}^{k} \frac{n(n-1) \ldots(n-j+1)\left(r ! / a_{r}\right)^{j}}{(r j+k) !} B_{r j+k, j}\left(0, \ldots, 0, a_{r+1}, a_{r+2}, \ldots\right) .
$$

We could use this theorem to prove that $S(n, n-k)$ is a polynomial in $n$ of degree $2 k$. In fact,

$$
S(n, n-k)=\sum_{j=0}^{k} S_{1}(2 k-j, k-j)\left(\begin{array}{c}
n \\
2 k-j
\end{array}\right)
$$

which is the principal reason the associated Stirling numbers were first defined.

We conclude this section by defining the special numbers which are related to $V(n, k)$.

The Bernoulli number $B_{n}$ is defined by means of

$$
x\left(e^{x}-1\right)^{-1}=\sum_{n=0}^{\infty} B_{n} x^{n} / n !, \quad \text { so } B_{n}=R_{n}(0,0,2,3,4, \ldots) .
$$

The Genocchi number $G_{n}$ is defined by means of

$$
2 x\left(e^{x}+1\right)^{-1}=\sum_{n=0}^{\infty} G_{n} x^{n} / n !, \quad \text { so } G_{n+1} /(n+1)=R_{n}(0,2,2,3,4, \ldots) \text {. }
$$

It is known [2, p. 49] that $G_{2 n}=2\left(1-2^{2 n}\right) B_{2 n}$.

The van der Pol number $V_{n}$ is defined by means of

$$
\begin{aligned}
\left(x^{3} / 6\right)\left[x\left(e^{x}+1\right)\right. & \left.-2\left(e^{x}-1\right)\right]^{-1} \\
= & \sum_{n=0}^{\infty} V_{n} x^{n} / n !, \quad \text { so } V_{n}=R_{n}(0,0,0,1,2,3, \ldots) .
\end{aligned}
$$

The van der Pol numbers have been the subject of several recent papers [8], [9] . They are closely related to the Rayleigh function of order $3 / 2$.

The number $A_{n}$ is defined by means of

(2.9) $\left(x^{2} / 2\right)\left(e^{x}-x-1\right)^{-1}=\sum_{n=0}^{\infty} A_{n} x_{n} / n !$, so $A_{n}=R_{n}(0,0,0,3,4,5, \ldots)$.

These numbers, which are similar to the Bernoulli numbers, have been examined in [3] and [6].

The number $W_{n}$ is defined by means of

$$
-4\left[x\left(e^{x}-1\right)-2\left(e^{x}+1\right)\right]^{-1}
$$

$$
=\sum_{n=0}^{\infty} W_{n} x^{n} / n !, \quad \text { so } W_{n}=R_{n}(-4,-2,0,1,2,3, \ldots)
$$


These numbers are discussed in [7] and [10] and are closely related to the Rayleigh function of order $-3 / 2$.

3. Properties of $V(n, k)$. Like all Bell polynomials, $V(n, k)$ has a fairly simple combinatorial interpretation. Consider a set partition of $\{1,2, \ldots, n\}$ into $k$ blocks, each block containing at least three elements. If a block has $m$ elements, assign it a "weight" of $m-2$. Then define the weight of the partition as the product of the weights of the blocks. It follows from $(1.1)$ that $V(n, k)$ is the sum of the weights of all the set partitions of $\{1,2, \ldots, n\}$ into $k$ blocks, each block having at least three elements. For example, there are $\left(\begin{array}{l}7 \\ 3\end{array}\right)=35$ set partitions of $\{1,2,3,4,5,6,7\}$ into two blocks, each block with at least three elements. Clearly, one block has three elements and the other has four elements, so each partition has weight 2 . Thus, $V(7,2)$ $=70$.

It is clear from this interpretation, or from (1.1), that $V(n, 0)=0$ if $n>0$, $V(n, 1)=n-2$ if $n>1, V(n, k)=0$ if $n<3 k$ and $V(3 k, k)=(3 k) ! 6^{-k} / k !$. Also, $V(0,0)=1$.

The following recurrence formula can be proved.

$$
2 V(n+1, k)=(k+n) V(n, k)-k n V(n-1, k)+n(n-1) V(n-2, k-1)
$$

To prove (3.1), define the polynomial $V_{n}(y)$ by means of

$$
\exp y\left[x\left(e^{x}+1\right)-2\left(e^{x}-1\right)\right]=\sum_{n=0}^{\infty} V_{n}(y) x^{n} / n !
$$

It follows that

$$
V_{n}(y)=\sum_{k=0}^{n} V(n, k) y^{k}
$$

Now take $y(x-1)$ times the partial derivative of (3.2) with respect to $y$ and subtract $(x-2)$ times the partial derivative of (3.2) with respect to $x$. Comparing coefficients of $x^{n} y^{k}$, we have (3.1). Using (3.1), we can compute the following values of $V(n, k)$.

\begin{tabular}{lllll}
$n / k$ & 1 & 2 & 3 & 4 \\
\hline 3 & 1 & & & \\
4 & 2 & & & \\
5 & 3 & & & \\
6 & 4 & 10 & & \\
7 & 5 & 70 & & \\
8 & 6 & 308 & & \\
9 & 7 & 1092 & 280 & \\
10 & 8 & 3414 & 4200 & \\
11 & 9 & 9834 & 36960 & \\
12 & 10 & 26752 & 249480 & 15400
\end{tabular}


Other recurrence formulas can be found. If we take the partial derivative of (3.2) with respect to $x$, subtract $y$ times the partial derivative of (3.2) with respect to $y$ and compare coefficients of $x^{n} y^{k}$, we have

$$
V(n+1, k)=k V(n, k)+\sum_{r=0}^{n-2}\left(\begin{array}{l}
n \\
r
\end{array}\right) V(r, k-1) .
$$

Also, from the partial derivative of (3.2) with respect to $x$,

$$
V(n+1, k)=\sum_{r=0}^{n-2}\left(\begin{array}{l}
n \\
r
\end{array}\right)(n-r-1) V(r, k-1) .
$$

It follows from (3.5) that $V(n, k)$ is a nonnegative integer. It also follows that, for $k>1, V(n, k)$ is even. The proof is by induction on $k$. If $k=2$, we have, by (3.5),

$$
V(n+1,2)=\sum_{r=0}^{n-5}\left(\begin{array}{c}
n \\
r+3
\end{array}\right)(n-r-4)(r+1)
$$

It is clear that if $n$ is even, each term on the right side of (3.6) is even. Suppose $n$ is odd: $n=1+2^{c} 1+\cdots+2^{c}$. Then $(n-r-4)(r+1)\left(\begin{array}{c}n \\ r+3\end{array}\right)$ is even unless $r+3$ $=1+e_{1} 2^{c} 1+\cdots+e_{s} 2^{c}$, each $e_{i}=0$ or 1 . Since $3 \leqslant r+3 \leqslant n-2$, we see there are $2^{s}-2$ (an even number) of odd numbers on the right side of (3.6). Thus, $V(n+1,2)$ is even, and it now follows easily from (3.5) that $V(n, k)$ is even for $k$ $\geqslant 2$.

We next define numbers $W(n, k)$ which are related to $V(n, k)$ in the same way the Stirling numbers are related to the associated Stirling numbers. Define $W(n, k)$ by means of

$$
k ! \sum_{n=0}^{\infty} W(n, k) x^{n} / n !=\left[(x-2)\left(e^{x}-1\right)\right]^{k}
$$

It follows that $W(1,1)=-2, W(n, 1)=n-2$ if $n>1, W(n, n)=(-2)^{n}$ and

$$
W(n+1, k)=-2 W(n, k-1)+\sum_{r=0}^{n-2}\left(\begin{array}{l}
n \\
r
\end{array}\right)(n-r-1) W(r, k-1) .
$$

It also follows from (3.7) that

$$
W(n, k)=\sum_{r=0}^{n} r !\left(\begin{array}{l}
n \\
r
\end{array}\right)\left(\begin{array}{l}
k \\
r
\end{array}\right)(-2)^{k-r} S(n-r, k),
$$

$$
S(n, k)=(-2)^{k} \sum_{r=0}^{n} r !\left(\begin{array}{c}
n \\
r
\end{array}\right)\left(\begin{array}{c}
r+k-1 \\
k-1
\end{array}\right) 2^{-r} W(n-r, k)
$$

where $S(n, k)$ is the Stirling number of the second kind. 
Using the same method we used for $V(n, k)$, we can derive the following recurrence:

$$
\begin{aligned}
2 W(n+1, k)= & (k+n) W(n, k)-4 W(n, k-1)-n k W(n-1, k) \\
& +4 n W(n-1, k-1)-n(n-1) W(n-2, k-1) .
\end{aligned}
$$

It follows that $W(n+1, n)=0$. Also, the following table of values for $W(n, k)$ can be constructed.

\begin{tabular}{ccccccc}
$n / k$ & 1 & 2 & 3 & 4 & 5 & 6 \\
\hline 1 & -2 & & & & & \\
2 & 0 & 4 & & & & \\
3 & 1 & 0 & -8 & & & \\
4 & 2 & -8 & 0 & 16 & & \\
5 & 3 & -20 & 40 & 0 & -32 & \\
6 & 4 & -26 & 120 & -160 & 0 & 64
\end{tabular}

It follows from (3.7) that $W(n, k)=B_{n, k}(-2,0,1,2,3, \ldots)$. Therefore, by Theorem 2.2, we have

$$
W(n, n-k)=\sum_{j=0}^{k}(-2)^{n-2 k+j}\left(\begin{array}{c}
n \\
2 k-j
\end{array}\right) V(2 k-j, k-j),
$$

which can be compared to (2.5). Since the summation in (3.11) really starts at $j=$ $[(k+1) / 2]$, we see that $W(n, n-k)$ is a polynomial in $n$ of degree $2 k-[(k+1) / 2]$. We also have $[2$, p. 136], [5]:

$$
\begin{gathered}
W(n, k)=\sum_{j=0}^{k}(-2)^{j}\left(\begin{array}{l}
n \\
j
\end{array}\right) V(n-j, k-j), \\
V(n, k)=\sum_{j=0}^{k} 2^{j}\left(\begin{array}{l}
n \\
j
\end{array}\right) W(n-j, k-j) .
\end{gathered}
$$

4. Relationship of $V(n, k)$ to Special Numbers. We now show how the numbers defined in Section 2 can be expressed in terms of $V(n, k)$. If we let

$$
A(n, k)=V(n, k)-n V(n-1, k) / 2,
$$

our main results are the following:

$$
\begin{gathered}
V_{n}=\sum_{j=0}^{n}(-6)^{j} n ! j !\left(\begin{array}{c}
n+1 \\
j+1
\end{array}\right) V(n+3 j, j) /(n+3 j) !, \\
B_{n}=\sum_{j=0}^{n-1} 2^{-j} A(n+j, j) /\left(\begin{array}{c}
n+j \\
j
\end{array}\right), \\
G_{n}=n \sum_{j=0}^{n-1} 4^{-j} j ! A(n-1, j),
\end{gathered}
$$




$$
A_{n}=\sum_{j=0}^{n} n ! j ! A(n+2 j, j) /(n+2 j) ! .
$$

Since $V(n, k)=B_{n, k}(0,0,1,2,3, \ldots)$ and $V_{n}$ is defined by (2.8), we see that (4.2) follows from Theorem 2.1. We note that also by Theorem 2.1 we have

$$
W_{n}=\sum_{j=1}^{n} 4^{-j} j ! W(n, j)
$$

where $W_{n}$ is defined by (2.10). Thus, by (3.9) and (4.6) we can express $W_{n}$ in terms of the Stirling numbers:

$$
W_{n}=\sum_{k=1}^{n} \sum_{r=0}^{n}(-2)^{-k-r} k ! r !\left(\begin{array}{l}
n \\
r
\end{array}\right)\left(\begin{array}{l}
k \\
r
\end{array}\right) S(n-r, k) .
$$

Now define $B_{n}^{\prime}$ by means of

$$
-2 x\left[(x-2)\left(e^{x}-1\right)\right]^{-1}=\sum_{n=0}^{\infty} B_{n}^{\prime} x^{n} / n ! .
$$

If we multiply both sides of (4.8) by $x-2$, it is easy to see that

$$
B_{n}=B_{n}^{\prime}-n B_{n-1}^{\prime} / 2,
$$

where $B_{n}$ is the Bernoulli number defined by (2.6). Also

$$
B_{n}^{\prime}=\sum_{r=0}^{n} 2^{r-n}(n)_{n-r} B_{r}
$$

which follows from the expansions of $x\left(e^{x}-1\right)^{-1}$ and $(x-2)^{-1}$ in (4.8). Since $B_{n}^{\prime}=R_{n}(0,-2,0,1,2,3, \ldots)$, we have, by Theorem 2.1,

$$
\begin{gathered}
B_{n}^{\prime}=\sum_{j=1}^{n} 2^{-j} V(n+j, j) /\left(\begin{array}{c}
n+j \\
j
\end{array}\right), \\
B_{n}^{\prime}=\sum_{j=1}^{n} 2^{-j}\left(\begin{array}{c}
n+1 \\
j+1
\end{array}\right) W(n+j, j) /\left(\begin{array}{c}
n+j \\
j
\end{array}\right) .
\end{gathered}
$$

Thus, if $A(n, k)$ is defined by (4.1), we see that (4.3) follows from (4.9) and (4.10). We proceed in the same way with the Genocchi numbers. Define $G_{n}^{\prime}$ by means of

$$
\begin{gathered}
-4\left[(x-2)\left(e^{x}+1\right)\right]^{-1}=\sum_{n=0}^{\infty} G_{n}^{\prime} x^{n} / n !, \text { so } \\
G_{n}=n G_{n-1}^{\prime}-\left(\begin{array}{c}
n \\
2
\end{array}\right) G_{n-2}^{\prime}, \quad G_{n}^{\prime}=\sum_{r=0}^{n} 2^{r-n}(n)_{n-r} G_{r} .
\end{gathered}
$$

Since $G_{n}^{\prime}=R_{n}(-4,0,0,1,2,3, \ldots)$, we have, by Theorem 2.1,

$$
G_{n}^{\prime}=\sum_{j=1}^{n} 4^{-j} j ! V(n, j)
$$


and (4.4) follows from (4.13) and (4.14). We note that (4.4) gives us, for $n>3$,

$$
2\left(1-2^{n}\right) B_{n}=n \sum_{j=1}^{n-1} 4^{-j} j ! A(n-1, j) .
$$

The upper limit in the sum can be replaced by $[(n-1) / 3]$.

Now define $A_{n}^{\prime}$ by means of

$$
\begin{aligned}
& -x^{2}\left[(x-2)\left(e^{x}-x-1\right)\right]^{-1}=\sum_{n=0}^{\infty} A_{n}^{\prime} x^{n} / n !, \text { so } \\
& A_{n}=A_{n}^{\prime}-n A_{n-1}^{\prime} / 2, \quad A_{n}^{\prime}=\sum_{r=0}^{n} 2^{r-n}(n)_{n-r} A_{r} .
\end{aligned}
$$

Since $A_{n}^{\prime}=R_{n}(0,0,-2,1,2,3, \ldots)$, we have, by Theorem 2.1,

$$
A_{n}^{\prime}=\sum_{j=1}^{n} n ! j ! V(n+2 j, j) /(n+2 j) !,
$$

and (4.5) follows from (4.17) and (4.18).

A generating function for $A(n, k)$ is

$$
(1-x / 2)\left[x\left(e^{x}+1\right)-2\left(e^{x}-1\right)\right]^{k}=k ! \sum_{n=3 k}^{\infty} A(n, k) x^{n} / n !,
$$

so we have

$$
V(n, k)=\sum_{r=3 k}^{n} 2^{r-n}(n)_{r} A(r, k) .
$$

Since $V(n, k)$ is an even integer for $k>1$, it follows from (4.1) that, except for $A(1,0)=-1 / 2, A(n, k)$ is an integer. Also, $A(n, 0)=0$ if $n>1, A(n, 1)=$ $(n-4)(1-n) / 2, A(n, k)=0$ if $n<3 k$. A small table of values for $A(n, k)$ follows.

\begin{tabular}{lclll}
$n / k$ & 1 & 2 & 3 & 4 \\
\hline 5 & -2 & & & \\
6 & -5 & 10 & & \\
7 & -9 & 35 & & \\
8 & -14 & 28 & & \\
9 & -20 & -294 & 280 & \\
10 & -27 & -2046 & 2800 & \\
11 & -35 & -8943 & 13860 & \\
12 & -44 & -32252 & 27720 & 15400
\end{tabular}

5. The Numbers $B_{n, k}\left(a_{1}, a_{2}, 3-\alpha, 4-\alpha, \ldots\right)$. We now look at the results of Sections 3 and 4 in a more general setting. It is convenient to use the following notation:

$$
B_{n, k}\left(a_{1}, a_{2} \mid \alpha\right)=B_{n, k}\left(a_{1}, a_{2}, 3-\alpha, 4-\alpha, 5-\alpha, \ldots\right)
$$




$$
R_{n}\left(a_{0}, a_{1}, a_{2} \mid \alpha\right)=R_{n}\left(a_{0}, a_{1}, a_{2}, 3-\alpha, 4-\alpha, \ldots\right) .
$$

Our main results in this section are Eqs. (5.11), (5.12) and (5.13) which generalize (4.3), (4.4) and (4.5).

There are many special cases of (5.1) and (5.2) that are of interest. For example,

$$
\begin{aligned}
& B_{n, k}(0,2 \mid 0)=n ! S(n-k, k) /(n-k) ! \\
& B_{n, k}(0,0 \mid 0)=n ! S_{1}(n-k, k) /(n-k) ! \\
& B_{n, k}(0,0 \mid 2)=V(n, k), \\
& B_{n, k}(-2,0 \mid 2)=W(n, k), \\
& B_{n, k}(1,2 \mid 0)=\left(\begin{array}{l}
n \\
k
\end{array}\right) k^{n-k}, \\
& B_{n, k}(x, 2 \mid 0)=\left(\begin{array}{l}
n \\
k
\end{array}\right) \sum_{r=0}^{k}\left(\begin{array}{l}
k \\
r
\end{array}\right)(k-r)^{n-k}(x-1)^{r}, \\
& B_{n, k}(0,1 \mid 1)=\sum_{r=0}^{n}(-1)^{n-r}\left(\begin{array}{l}
n \\
r
\end{array}\right) k^{r} S_{1}(n-r, k), \\
& R_{n}(0,0,0 \mid 0)=A_{n}, \\
& R_{n}(0,0,2 \mid 0)=B_{n}, \\
& R_{n}(0,1,2 \mid 0)=(-1)^{n}, \\
& R_{n}(0,2,2 \mid 0)=G_{n+1} /(n+1), \\
& R_{n}(0,0,0 \mid 2)=V_{n}, \\
& R_{n}(-4,-2,0 \mid 2)=W_{n},
\end{aligned}
$$

$R_{n}(0,1-\lambda, 210)=H_{n}(\lambda)$, a function related to the Eulerian numbers [1], $R_{n}(-1,0,1 \mid 1)=d_{n}$, the number of derangements of $n$.

By Theorem 2.1 we have

$$
\begin{gathered}
R_{n}\left(a_{0}, a_{1}, a_{2} \mid \alpha\right)=\sum_{j=1}^{n}\left(-a_{0}\right)^{-j} j ! B_{n, j}\left(a_{1}, a_{2} \mid \alpha\right), \\
R_{n}\left(0, a_{1}, a_{2} \mid \alpha\right)=\sum_{j=1}^{n}\left(-a_{1}\right)^{-j} B_{n+j, j}\left(0, a_{2} \mid \alpha\right) /\left(\begin{array}{c}
n+j \\
j
\end{array}\right) \\
=\sum_{j=1}^{n}\left(-a_{1}\right)^{-j}\left(\begin{array}{c}
n+1 \\
j+1
\end{array}\right) B_{n+j, j}\left(a_{1}, a_{2} \mid \alpha\right) /\left(\begin{array}{c}
n+j \\
j
\end{array}\right),
\end{gathered}
$$




$$
\begin{aligned}
& R_{n}\left(0,0, a_{2} \mid \alpha\right)=\sum_{j=1}^{n}\left(-a_{2}\right)^{-j} 2^{j} n ! j ! B_{n+2 j, j}(0,0 \mid \alpha) /(n+2 j) ! \\
& =\sum_{j=1}^{n}\left(-a_{2}\right)^{-j} 2^{j} n ! j !\left(\begin{array}{c}
n+1 \\
j+1
\end{array}\right) B_{n+2 j, j}\left(0, a_{2} \mid \alpha\right) /(n+2 j) !, \\
& R_{n}(0,0,0 \mid \alpha)=\sum_{j=1}^{n}(\alpha-3)^{-j} n ! j ! 6^{j}\left(\begin{array}{c}
n+1 \\
j+1
\end{array}\right) B_{n+3 j, j}(0,0 \mid \alpha) /(n+3 j) !
\end{aligned}
$$

$$
\text { for } \alpha \neq 3 \text {. }
$$

We can now generalize the results of Section 4. Using the same reasoning we used for $\alpha=2$, we see that for $\alpha \neq 0$,

(5.10) $(-1)^{n}=R_{n}(-\alpha, 1-\alpha, 2-\alpha \mid \alpha)-n R_{n-1}(-\alpha, 1-\alpha, 2-\alpha \mid \alpha) / \alpha$.

Letting $A_{n, k}^{\prime}\left(a_{1}, a_{2} \mid \alpha\right)=B_{n, k}\left(a_{1}, a_{2} \mid \alpha\right)-n B_{n-1, k}\left(a_{1}, a_{2} \mid \alpha\right) / \alpha$ and combining (5.3)(5.5) and (5.7)-(5.10), we have

$$
\begin{aligned}
B_{n} & =\sum_{j=1}^{n} \alpha^{-j} A_{n+j, j}^{\prime}(0,2-\alpha \mid \alpha) /\left(\begin{array}{c}
n+j \\
j
\end{array}\right), \\
G_{n} & =\sum_{j=0}^{n-1} n(2 \alpha)^{-j} j ! A_{n-1, j}^{\prime}(2-\alpha, 2-\alpha \mid \alpha), \\
A_{n} & =\sum_{j=0}^{n} 2^{j} \alpha^{-j} n ! j ! A_{n+2 j, j}^{\prime}(0,0 \mid \alpha) /(n+2 j) !, \\
(-1)^{n} & =\sum_{j=0}^{n} \alpha^{-j} j ! A_{n, j}^{\prime}(1-\alpha, 2-\alpha \mid \alpha) .
\end{aligned}
$$

Thus (5.6), (5.11), (5.12) and (5.13) generalize (4.2)-(4.5).

By Theorem 2.2 we have

$$
\begin{aligned}
& B_{n+k, n}\left(a_{1}, a_{2} \mid \alpha\right)=\sum_{j=1}^{k} a_{1}^{n-j}\left(\begin{array}{c}
n+k \\
j+k
\end{array}\right) B_{j+k, j}\left(0, a_{2} \mid \alpha\right) \\
& B_{2 n+k, n}\left(0, a_{2} \mid \alpha\right)=\sum_{j=1}^{k}\left(\frac{a_{2}}{2}\right)^{n-j} \frac{(2 n+k) !}{(n-j) !(2 j+k) !} B_{2 j+k, j}(0,0 \mid \alpha),
\end{aligned}
$$

and there are other similar formulas, of which (3.12) and (3.13) are special cases [5].

Using exactly the same method we used to derive (3.1), we can find a recurrence formula for $B_{n, k}\left(a_{1}, a_{2} \mid \alpha\right)$. Letting $B_{n, k}=B_{n, k}\left(a_{1}, a_{2} \mid \alpha\right)$, we have

$$
\begin{aligned}
\alpha B_{n+1, k}= & {[n+(\alpha-1) k] B_{n, k}-k n B_{n-1, k}+c_{0} B_{n, k-1}+n c_{1} B_{n-1, k-1} } \\
& +n(n-1) c_{2} B_{n-2, k-1}+n(n-1)(n-2) c_{3} B_{n-3, k-1},
\end{aligned}
$$


where

$$
\begin{aligned}
& c_{0}=a_{1} \alpha, \\
& c_{1}=\alpha\left(a_{2}-a_{1}\right), \\
& c_{2}=-\alpha^{2} / 2+\left(3-a_{2}\right) \alpha / 2+a_{1}-a_{2} / 2, \\
& c_{3}=\alpha / 2-1+a_{2} / 2 .
\end{aligned}
$$

Also, $B_{0,0}=1, B_{n, 0}=0$ if $n>0, B_{n, 1}=n-\alpha$ if $n>2, B_{n, k}=0$ if $n<k$.

Finally, as an application of formulas (5.3)-(5.6), we show how some of the formulas in [2], [10] and [11] involving Bernoulli and Stirling numbers can be proved. Using the special values of $B_{n, k}\left(a_{1}, a_{2} \mid \alpha\right)$ and $R_{n}\left(a_{0}, a_{1}, a_{2} \mid \alpha\right)$ listed earlier, we have, from (5.3)-(5.6) and (5.15),

$$
\begin{array}{rlr}
(-1)^{n} & =\sum_{j=1}^{n}(-1)^{j} j ! S(n, j) & {[10, \mathrm{p} .170]} \\
B_{n}=\sum_{j=1}^{n}(-1)^{j}\left(\begin{array}{c}
n+1 \\
j+1
\end{array}\right) S(n+j, j) /\left(\begin{array}{c}
n+j \\
j
\end{array}\right) & {[10, \mathrm{p} .219]} \\
B_{n}=\sum_{j=1}^{n}(-1)^{j} S_{1}(n, j) /\left(\begin{array}{c}
n+j \\
j
\end{array}\right) & {[10, \text { p. 599] }}
\end{array}
$$

$$
\sum_{j=0}^{n} n ! S(k, n-j) x^{j} / j !=\sum_{j=0}^{n}\left(\begin{array}{c}
n \\
j
\end{array}\right)(n-j)^{k}(x-1)^{j}
$$

$$
S(n, k)=\sum_{j=1}^{k}(-1)^{j}\left(\begin{array}{l}
k \\
j
\end{array}\right)(k-j)^{n} / k ! \quad[2, \text { p. 206] }
$$

$$
k ! S(n, k)=k^{n}-\sum_{j=1}^{k-1}(k)_{j} S(n, j) \quad[2, \text { p. 209] }
$$

$$
G_{n+1}=\sum_{j=1}^{n}(-2)^{-j} j !(n+1) S(n, j)
$$

$$
\begin{gathered}
H_{n}(\lambda)=\sum_{j=1}^{n} j !(\lambda-1)^{-j} S(n, j), \quad(\lambda \neq 1), \\
A_{n}=\sum_{j=1}^{n}(-1)^{j}\left(\begin{array}{c}
n+1 \\
j+1
\end{array}\right) S_{1}(n+2 j, j) /\left(\begin{array}{c}
n+2 j \\
n
\end{array}\right)[1.3 \ldots(2 j-1)]
\end{gathered}
$$

6. Inverse Array for $W(n, k)$. If $W(n, k)$ is defined by (3.7), it is possible to find an inverse array $(w(n, k))(n=0,1,2, \ldots ; k=0,1, \ldots, n)$ such that

$$
\sum_{k=j}^{n}(-2)^{-k} W(n, k) w(k, j)=\delta_{n, j}=\sum_{k=j}^{n} w(n, k)(-2)^{-j} W(k, j)
$$


By using a method described by L. Carlitz ("A special class of triangular arrays," Collect. Math., v. 27, 1976, pp. 23-58), we shall prove

$$
w(n, k)=\sum_{r=0}^{n-k}\left(\begin{array}{c}
n+r-1 \\
r
\end{array}\right) \frac{(k+r-1) !}{(k-1) !} 2^{-r} s(n, k+r),
$$

where $s(n, k+r)$ is the signed Stirling number of the first kind [2, p. 212]. The author wishes to thank Professor Carlitz for his suggestions concerning this section.

We begin by defining $f(z)=(1-z / 2)\left(e^{z}-1\right)$, so that

$$
\exp (x f(z))=1+\sum_{n=1}^{\infty} \frac{z^{n}}{n !} \sum_{k=1}^{n}(-2)^{-k} W(n, k) x^{k}
$$

If $g(z)$ is the inverse of $f(z)$, i.e. $f(g(z))=g(f(z))=z$, we define $w(n, k)$ by means of

$$
\exp (x g(z))=1+\sum_{n=1}^{\infty} \frac{z^{n}}{n !} \sum_{k=1}^{n} w(n, k) x^{k}
$$

and by the results in the Carlitz paper mentioned above, (6.1) follows.

We can find $g(z)$ and, more generally, $g^{k}(z)$ by means of the Lagrange inversion formula $[2$, p. 148]. We have

$$
g^{k}(z)=\sum_{n=1}^{\infty} \frac{z^{n}}{n !}\left[k(n-1) ! \frac{d^{n-k}}{d x^{n-k}}\left((1-x / 2)^{-1} \frac{x}{e^{x}-1}\right)^{n}\right] x=0
$$

and from the expansions

$$
\begin{gathered}
(1-x / 2)^{-n}=\sum_{r=0}^{\infty}\left(\begin{array}{c}
n+r-1 \\
r
\end{array}\right) 2^{-r} x^{r} \\
\left(\frac{x}{e^{x}-1}\right)^{n}=\sum_{i=0}^{\infty} B_{i}^{(n)} x^{i} / i !=\sum_{i=0}^{\infty}\left(\begin{array}{c}
n-1 \\
i
\end{array}\right)^{-1} s(n, n-i) x^{i} / i !
\end{gathered}
$$

$[2$, p. 228], we have

$$
g^{k}(z)=\sum_{n=1}^{\infty} \frac{z^{n}}{n !}\left[k \sum_{r=0}^{n-k}\left(\begin{array}{c}
n+r-1 \\
r
\end{array}\right)(k+r-1) ! 2^{-r} s(n, k+r)\right] .
$$

Formula (6.2) now follows from (6.3) and (6.4).

It follows from (6.2) that $w(n, n-k)$ is a polynomial in $n$ of degree $2 k$. We can write

$$
w(n, n-k)=\sum_{j=0}^{2 k} w^{\prime}(k, j)\left(\begin{array}{c}
n \\
2 k-j
\end{array}\right)
$$

though it appears difficult to find a generating function for $w^{\prime}(n, k)$. It also appears difficult to find a recurrence for $w(n, k)$. A small table of values for $w(n, k)$ follows. 


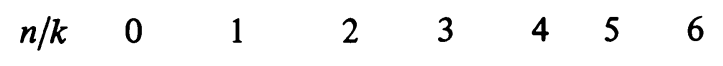

\begin{tabular}{cccccccc}
\hline 0 & 1 & & & & & & \\
1 & & 1 & & & & & \\
2 & & 0 & 1 & & & & \\
3 & & $1 / 2$ & 0 & 1 & & & \\
4 & & 1 & 2 & 0 & 1 & & \\
5 & & 4 & 5 & 5 & 0 & 1 & \\
6 & & $39 / 2$ & $53 / 2$ & 15 & 10 & 0 & 1
\end{tabular}

From (6.1) we have $w(n, n)=1, w(n, n-1)=0, w(n, n-2)=$ $(-1)^{n-1} 2^{2-n} W(n, n-2), w(n, n-3)=(-1)^{n} 2^{3-n} W(n, n-3)$.

Department of Mathematics

Wake Forest University

Winston-Salem, North Carolina 27109

260.

1. L. CARLITZ, "Eulerian numbers and polynomials," Math. Mag., v. 33, 1959, pp. 247-

2. L. COMTET, Advanced Combinatorics, Reidel, Dordrecht and Boston, Mass., 1974.

3. F. T. HOWARD, "A sequence of numbers related to the exponential function," Duke Math. J., v. 34, 1967, pp. 599-616.

4. F. T. HOWARD, "Associated Stirling numbers," Fibonacci Quart. (To appear.)

5. F. T. HOWARD, "Bell polynomials and degenerate Stirling numbers," Rend. Sem. Mat. Padova. (To appear.)

6. F. T. HOWARD, "Numbers generated by the reciprocal of $e^{x}-x-1$," Math. Comp., v. 31, 1977, pp. 581-598.

7. F. T. HOWARD, "Polynomials related to the Bessel functions," Trans. Amer. Math. Soc., v. 210, 1975, pp. 233-248.

8. F. T. HOWARD, "Properties of the van der Pol numbers and polynomials," J. Reine Angew. Math., v. 260, 1973, pp. 35-46.

9. F. T. HOWARD, "The van der Pol numbers and a related sequence of rational numbers," Math. Nachr., v. 42, 1969, pp. 80-102.

10. C. JORDAN, Calculus of Finite Differences, Chelsea, New York, 1950.

11. J. RIORDAN, An Introduction to Combinatorial Analysis, Wiley, New York, 1958. 